\title{
Long-term EEG monitoring and positron emission tomography in evaluating patients with drug-resistant epilepsy
}

\author{
Abdelmoneim Ahmed Nagy Eissa', Wafik Said Bahnasy ${ }^{1 *}$ (D), Al Siagy Ali Abd Elaziz Salama², \\ Elsayed Ali Mohamed Tag Eldin ${ }^{1}$ and Hazem Abdelrahman Fayed ${ }^{1}$
}

\begin{abstract}
Background: Drug-resistant epilepsy (DRE) is a commonly faced problem in epilepsy practice associated with great psychosocial consequences. This work aimed to study the role of seizure semiology analysis (SSA), long-term EEG monitoring, high field strength MRI, and positron emission tomography (PET) scan of the brain to confirm the diagnosis of true-RDE cases as well as seizure onset zone (SOZ) localization.

Methods: This study was conducted on 67 DRE patients subjected to SSA, long-term video EEG, 3-Tesla MRI brain, and 18-FDG PET scan of brain.

Results: Forty-three patients had true-DRE and 24 had non-epileptic episodic events (NEEE). The former group consisted of 23 patients with temporal lobe, 17 with frontal lobe, and 3 with occipital lobe epilepsies. Forty-eight patients had abnormal EEG included all patients with true-DRE and 5 patients with NEEE. Thirty patients had abnormal MRI brain findings in the form of mesial temporal sclerosis and/or focal cortical dysplasia. Sixteen true-DRE patients with normal 3-T MRI underwent PET scan of the brain where regional hypometabolism was detected in 14 (87.5\%) of them.

Conclusion: Practicing with DRE patients' needs meticulous assessment through detailed SSA, adequate neurophysiological long-term video EEG monitoring, and thorough neuroradiological investigation (structural and/or functional) to confirm the diagnosis of true-DRE and localize the SOZ as well as identification of patients with NEEE.
\end{abstract}

Keywords: Drug-resistant epilepsy, Paroxysmal non-epileptic events, Mesial temporal sclerosis, Long-term EEG, PET scan

\section{Introduction}

Drug-resistant epilepsy (DRE) is defined as the failure of adequate trials with two tolerated, appropriately chosen and used antiepileptic drugs (AEDs) schedules (whether as monotherapies or in combination) to achieve sustained seizure freedom [1]. DRE constitutes about $20-40 \%$ of epileptic patients which is associated with poor prognostic consequences including SUDEP (sudden unexpected death in epilepsy), physical injury, psychosocial dysfunction, increased economic burden, and reduced quality of

\footnotetext{
* Correspondence: wafiq.elbahnasi@med.tanta.edu.eg

The work was carried out in The Epilepsy Clinics, Neuropsychiatry

Department and Center of Neurology and Psychiatry, Tanta University

Hospitals, Faculty of Medicine, Tanta University, zip code 31527, Tanta, Egypt.

'Department of Neuropsychiatry, Faculty of Medicine, Tanta University, 31527

Tanta, Egypt

Full list of author information is available at the end of the article
}

life [2]. Assessment of DRE is challenging to avoid faulty diagnosis of patients with non-epileptic episodic events (NEEE) as having true seizure intractability [3]. The NEEE may be caused by paroxysmal disorders of non-epileptic nature including syncope, paroxysmal arrhythmias, parasomnias, and TIAs or psychogenic non-epileptic seizures (PNES) which is a functional neurological disorder characterized by behavioral or physical manifestation of a somatoform, conversion, or dissociative disorder $[4,5]$.

Non-invasive pre-surgical precise epileptogenic zone localization (EZL) is a crucial first step for accurate management DRE patients which is sometimes difficult with short-term EEG recording and low field strength MRI machines especially when lacking epilepsy imaging protocols [6]. Long-term EEG monitoring is highly beneficial in 
localization of the seizure onset zone (SOZ) in true-DRE patients as well as identification of NEEE cases [7]. At the same time, high field strength MRI with dedicated epilepsy protocols as well as interictal 18-fluorodeoxyglucose PET (18-FDG-PET) scan is highly valuable in EZL of patients with partial-onset seizures $[8,9]$.

\section{Aim of the work}

The aim of the work was to study the role of long-term video EEG monitoring, 3-T MRI, and 18-FDG-PET imaging in evaluating DRE patients with non-conclusive short-term EEG as well as 1.5-T brain MRI.

\section{Methods}

This paper is adopted from the thesis with the same title submitted by the first author to the Faculty of Medicine, Tanta University, in partial fulfillment of the MD degree in Neurology [10]. The work was an observational prospective cohort study conducted on 86 consecutive DRE patients with non-lesional 1.5-T MRI and normal 20min EEG recording. The patients were recruited from the epilepsy clinic of The Neuropsychiatry Department, Tanta University Hospitals in the period from the 1st of December 2015 to the end of November 2017. Nineteen patients were dropped out during follow-up and the remaining 67 DRE patients continued this phase of the study till its final steps. DRE was diagnosed according to the criteria of the international league against epilepsy (ILAE) 2010 [11].

Exclusion criteria comprised patients with definite 1.5-T MRI brain structural abnormalities, AEDs poor compliance as well as MRI or PET scan contraindications. The study's protocol was approved by The Research Ethics Committee and Quality Assurance Unit, Faculty of Medicine, Tanta University (approval code: 30564/11/15). Participation was voluntary, informed consents were approved by all contributors, and any possible risks were clarified.

Included subjects were submitted to history taking and available AEDs serum levels to check patients' compliance as well as seizure semiology analysis (SSA) according to Elwan and colleagues [12]. The analysis was performed blindly by 2 neurologists who were not aware of the original pre-assessment diagnosis. They considered ambiguous semiology (like frequent blinking, side to side movement of the head, intense rotation in bed, opisthotonos position, pelvic thrusting, hyperventilation, tip tongue biting, and/or crying) to be in favor of PNEE. Psychiatric assessment was performed using the Hamilton Anxiety Scale (HAS) and the Montgomery-Åsberg Depression Rating Scale (MADRS) for anxiety and depressive symptoms severities respectively. Patients were also subjected to 6-24-h long-term EEG video recording using a Nihon Kohden EEG system (model EEG1200, 64 channels, Japan) according to the guidelines of the American Clinical Neurophysiology Society [13]. The model has the opportunity of polysomnographic (PSG) assessment for patients with nocturnal paroxysmal hyperkinetic events. REM behavior disorders (RBD) was defined according to the International Classification of Sleep Disorders-III (ICSD-III) [14] as repeated episodes of sleep-related vocalization and/ or complex motor behaviors, documented by PSG to occur during REM sleep and the disturbance is not better explained by another sleep disorder, mental disorder, medication, or substance abuse.

Brain MRI was done using a 3-T MRI machine, Philips Medical Systems (Philips MR Ingenia Elition, 2016, Netherlands), with quadrature 16 channels head coil and following the epilepsy imaging protocol of Cendes and colleagues [15]. PET/CT scan was performed interictally by Philips Medical Systems (Philips Ingenuity TF PET/ CT, Model NO. 882442, 2015, Netherlands) according to the European Association of Nuclear Medicine Neuroimaging Committee guidelines for PET brain imaging using 18FDG, version 2 [16].

Statistical analysis was conducted using SPSS Prism, version 20, 2013 created by IBM, Illinois, Chicago, USA. Statistical differences between the studied groups were tested using chi-square for categorical variables and $t$ test for numerical ones. $p$ values $<0.05$ were considered statistically significant.

\section{Results}

The results of SSA of the included patients revealed that; 43 had true-DRE semiology (group-I) and 24 had NEEE (group-II). Group-II with NEEE comprised of 9 patients with PNES and 10 cases with NEEE due to physiological causes (4 syncopal attacks, 2 paroxysmal vertigo, 1 episodic migraine with aura, and 3 RBD) and 5 patients had history of controlled epilepsy with recent-onset PNES which incorporate them to the sector of intractability. Patients with true-DRE showed statistically significant increase in each of disease duration, family history of epilepsy, and history of febrile convulsion than patients with NEEE. At the same time, there were statistically significant decreases in each of seizure frequencies per month and severities of depressive as well as anxiety symptoms in true-DRE than NEEE patients (Table 1).

Regarding SSA of true-DRE patients; 23 had temporal lobe epilepsy (TLE), 15 had frontal lobe epilepsy (FLE), 3 had occipital lobe epilepsy (OLE), and 2 had insular lobe epilepsy manifestations. The TLE subgroup showed a statistically significant increase in each of the history of febrile convulsions and mean duration of seizure attacks compared to the FLE subgroup. At the same time, there were statistically significant decreases in each of the seizure frequency per month and frequency of nocturnal attacks in TLE compared to FLE subgroups (Table 2).

Regarding long-term EEG interpretation, interictal abnormalities were detected in 48 cases, $43(100 \%)$ of 
Table 1 Comparison between patients with true drug-resistant epilepsy (group I) and those with non-epileptic episodic events (group II) regarding clinical data

\begin{tabular}{|c|c|c|c|}
\hline & $\begin{array}{l}\text { Group I } \\
\text { (n 43) }\end{array}$ & $\begin{array}{l}\text { Group II } \\
\text { (n 24) }\end{array}$ & $p$ value \\
\hline Age (years) & $23.05 \pm 5.42$ & $21.7 \pm 4.84$ & 0.314 \\
\hline Male & $19(63.3 \%)$ & $11(36.7 \%)$ & 0.897 \\
\hline Female & $24(64.9 \%)$ & $13(35.1 \%)$ & \\
\hline Age of onset (years) & $13.13 \pm 3.68$ & $14.53 \pm 4.12$ & 0.157 \\
\hline Duration of illness (years) & $10.92 \pm 4.85$ & $6.17 \pm 3.12$ & $0.001^{*}$ \\
\hline Family history of epilepsy & $12(27.9 \%)$ & $3(12.5 \%)$ & $0.02^{*}$ \\
\hline History of febrile convulsions & 17 (39.5\%) & $2(8.3 \%)$ & $0.007^{*}$ \\
\hline Seizure frequency (per month) & $10 \pm 3.54$ & $15 \pm 4.58$ & $<0.0001^{*}$ \\
\hline Number of AEDs taken & $10.92 \pm 4.85$ & $6.17 \pm 3.12$ & $<0.0001^{*}$ \\
\hline Depressive symptoms (MADRS) & $11 \pm 1.83$ & $17 \pm 2.51$ & $<0.0001^{*}$ \\
\hline Anxiety symptoms (HAS) & $13.41 \pm 2.38$ & $26 \pm 4.72$ & $<0.0001^{*}$ \\
\hline
\end{tabular}

*Significant. AEDs: antiepileptic drugs, HAS: Hamilton Anxiety Scale, MADRS: Montgomery-Åsberg Depression Rating Scale

true-DRE and 5 (20.8\%) of NEEE patients. The abnormalities were either spike and wave, sharp and slow-wave, slow-spike and wave, poly-spike and wave, multiple sharp and slow waves, focal paroxysmal polymorphic delta slowing and/or multiple sharp complexes. Nine (37.5\%) cases with NEEE developed ictal events during the EEG recording which was significantly higher than those that occurred in true-DRE patients $(11.6 \%)$ with $p$ value $=0.01$.

The results of 3-T brain MRI revealed 37 patients with indeterminate structural lesions (16 patients with trueDRE and 21 cases with NEEE) and 30 cases with structural brain abnormalities ( 28 patients with true-DRE and
2 cases with NEEE). The abnormalities in the true-DRE patients included 14 cases with mesial temporal sclerosis (MTS) (either unilateral or bilateral), 12 with focal cortical dysplasia (FCD) (3 temporal, 9 frontal, and 1 occipital), and 2 cases with small arteriovenous malformations (Figs. 1 and 2). The 2 cases with NEEE MRI abnormalities had left temporal pole atrophy in one and frontal lobe encephalomalacia in the other.

Interictal 18-FDG-PET were performed to the 16 trueDRE patients with normal 3-T MRI. PET scan showed abnormal regional hypometabolism in 14 (87.5\%) cases; 5 had unilateral temporal, 2 had bitemporal, 3 had unilateral frontal, 3 had fronto-parietal, and one had occipital reduced tracer avidity (Fig. 3).

The study showed that EEG abnormalities were detected among patients with true-DRE; focal temporal epileptic activities were found among $44 \%$ of patients while focal frontal epileptic activities were found among $32.5 \%$ of patients. Multifocal epileptic activities were found among $16.5 \%$ of patients; lastly, focal occipital epileptic activities represented 7\% of patients. These EEG findings were concordant with seizure semiology in $84.2 \%$ of TLE and $92.8 \%$ of FLE.

The concordance of long-term EEG and MRI brain abnormalities was present in $73.34 \%$ of MTS patients and $53.84 \%$ of those with FLE. Regarding the correlation of long-term EEG results with that of 18-FDG-PET scan; $85.71 \%$ of TLE patients showed concordant abnormalities in both procedures compared to $60 \%$ of those with FLE.

\section{Discussion}

Failure of $\geq 2$ AEDs trials is a commonly faced problem in epilepsy practice which needs meticulous assessment

Table 2 Comparison between true drug-resistant epilepsy patients of temporal origin (group la) and frontal seizure onset zones (group Ib)

\begin{tabular}{|c|c|c|c|c|}
\hline & & $\begin{array}{l}\text { Group la } \\
(n \text { 23) }\end{array}$ & $\begin{array}{l}\text { Group Ib } \\
(n \text { 15) }\end{array}$ & $p$ value \\
\hline \multicolumn{2}{|c|}{ Age (years) } & $22.63 \pm 3.27$ & $24.12 \pm 4.25$ & 0.357 \\
\hline \multirow[t]{2}{*}{ Sex } & Male & $10(43.5 \%)$ & $8(47 \%)$ & 0.903 \\
\hline & Female & $13(56.5 \%)$ & $9(53 \%)$ & \\
\hline \multicolumn{2}{|c|}{ Age of onset (years) } & $12.72 \pm 2.47$ & $13.48 \pm 3.12$ & 0.418 \\
\hline \multicolumn{2}{|c|}{ Duration of illness (years) } & $9.91 \pm 2.45$ & $11.25 \pm 2.37$ & 0.174 \\
\hline \multicolumn{2}{|c|}{ Family history of epilepsy } & $9(39.1 \%)$ & $3(17.6 \%)$ & 0.175 \\
\hline \multicolumn{2}{|c|}{ History of febrile convulsions } & $13(56.5 \%)$ & $3(17.6 \%)$ & $0.044^{*}$ \\
\hline \multicolumn{2}{|c|}{ Seizure frequency (per month) } & $8.12 \pm 2.14$ & $12.37 \pm 1.85$ & $0.007^{*}$ \\
\hline \multicolumn{2}{|c|}{ Number of AEDs taken } & $4.14 \pm 1.87$ & $3.74 \pm 1.74$ & 0.437 \\
\hline \multicolumn{2}{|c|}{ Nocturnal seizure attacks } & $6(26.1 \%)$ & $11(73.3 \%)$ & $0.017^{*}$ \\
\hline \multicolumn{2}{|c|}{ Duration of seizures attacks (seconds) } & $317.17 \pm 144.48$ & $27.65 \pm 14.49$ & $0.001^{*}$ \\
\hline \multicolumn{2}{|l|}{ Aura } & $20(87 \%)$ & $10(66.7 \%)$ & 0,134 \\
\hline \multicolumn{2}{|c|}{ Automatism } & $20(87 \%)$ & $11(73.3 \%)$ & 0.201 \\
\hline
\end{tabular}

*Significant. AEDs: antiepileptic drugs 


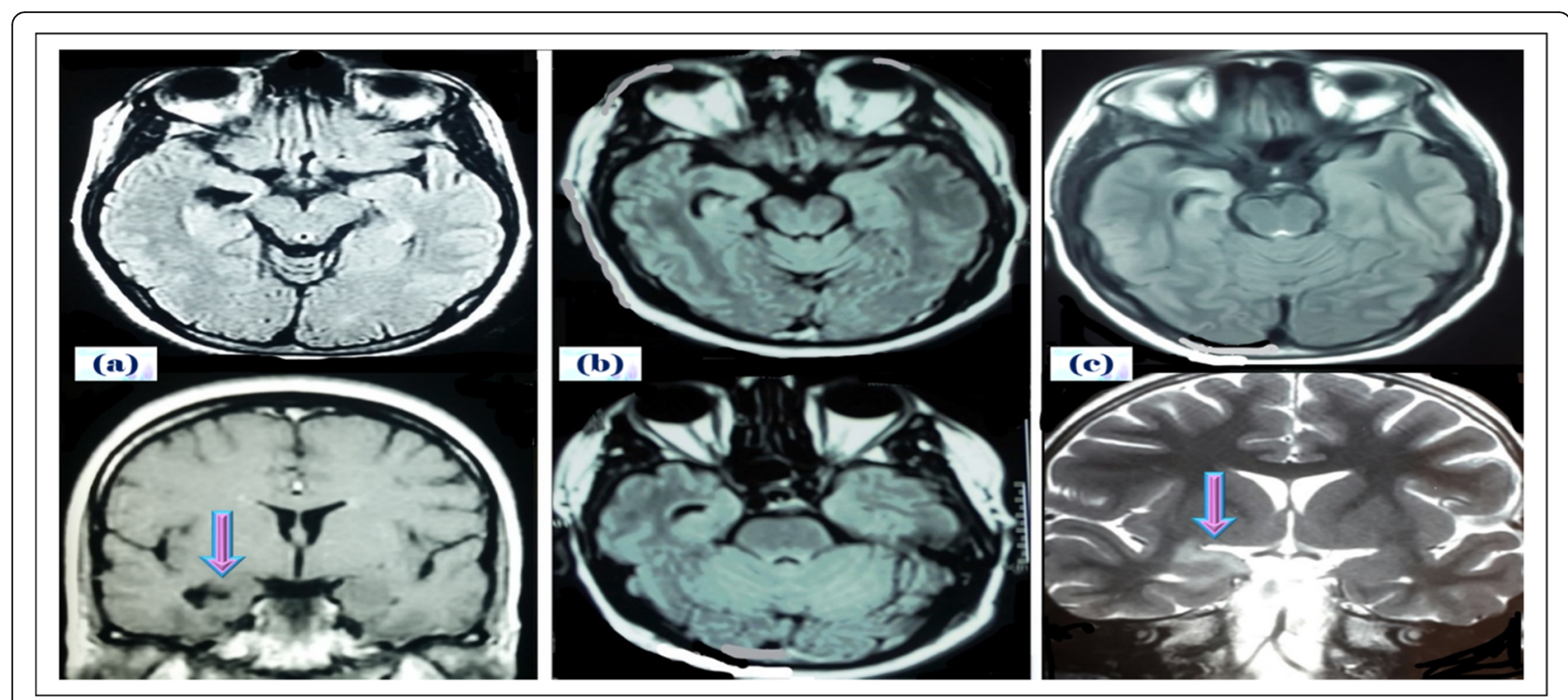

Fig. 1 Magnetic resonance imaging of 3 drug-resistant epilepsy cases (a, b, and $\mathbf{c}$ ) with right mesial temporal sclerosis

of patients for proper management [17]. The current study showed nearly one-third of originally diagnosed DRE patients in the epilepsy clinic had NEEE due toimprecise initial diagnosis of functional seizures because of SSA and/or EEG misinterpretation as well as faulty diagnosis of frequent pseudo-seizures occurring in controlled epileptic patients relied on the previous organic epileptic nature of their illnesses. Any initial misdiagnosis was followed by focusing on reduction of the ictus frequency rather than reanalysis and revision of the non-organic disease with consecutive unnecessary increase of AEDs and pushing the patients to the corner of seizure intractability. The most common NEEE were PNES followed by syncope, REM sleep behavior disorder, paroxysmal vertigo, and migraine. These results are in accordance with that of Wilkins and colleagues [18] as well as Hanrahan and colleagues [19] who claimed diagnostic delay of NEEE to faulty baseline epilepsy diagnosis as
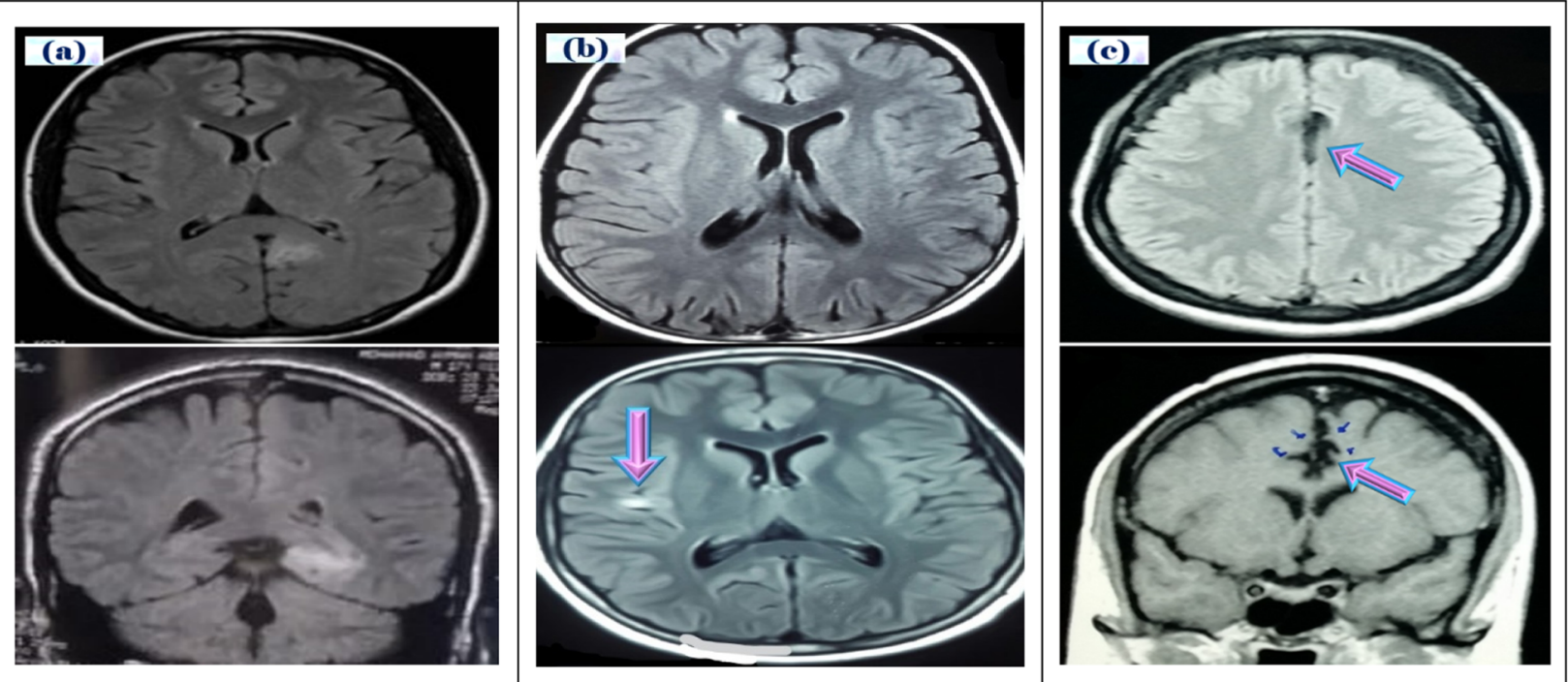

Fig. 2 Magnetic resonance imaging of 3 included cases. a Left limbic lobe focal cortical dysplasia. b Right insular lobe focal cortical dysplasia. c Falicine arteriovenous malformation with bilateral medial frontal cortex encroachment 


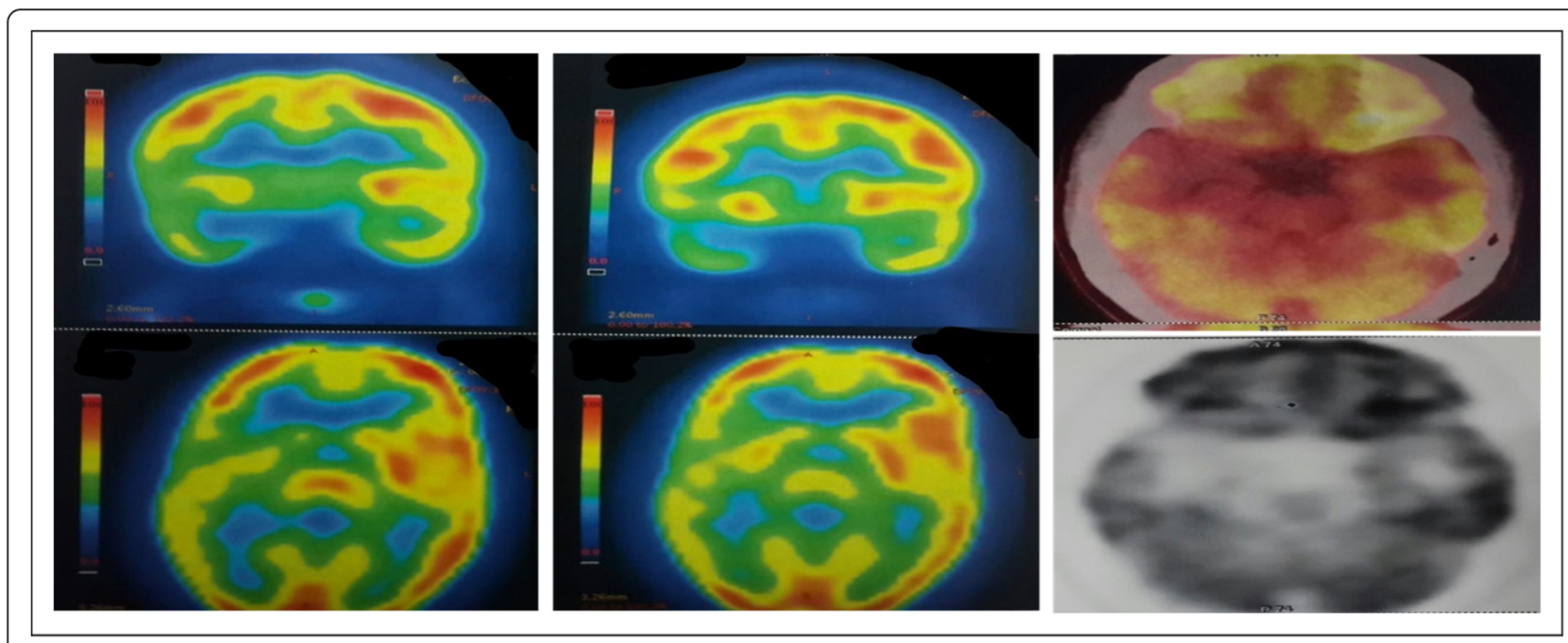

Fig. 3 18-FDG-PET/CT scan showing interictal hypometabolism with reduced tracer avidity of the right medial temporal region

well as difficulty in diagnosis of PNES in epileptic patients with successive multiple trials to reduce seizure frequencies by increasing the AEDs.

The study showed that female sex, high seizure frequencies, shorter disease duration, and multiple or ambiguous semiology as well as prominent depressive and anxiety symptoms are in favor of PNES. The current results were concordant with that of Korucuk and colleagues [20] as well as Labate and colleagues [21] who stated that distinguishing PNES from true epileptic ones may be difficult even to experienced observers which must be suspected in any epileptic case with recurrent paroxysmal behavioral abnormalities. They also stated that the evolution of epilepsy monitoring units or the ability to utilize simultaneous video and long-term EEG recordings may be a key to diagnosis. PNES could be classified to generalized motor seizures, akinetic seizures, seizures with subjective symptoms, and focal motor seizures.

The current results revealed that family history of epilepsy, history of febrile convulsions, single seizure semiology, presence of aura, automatism, and nocturnal seizures are in accord with true-DRE. These results are concordant with the studies of Hintz and colleagues [22] as well as Guevara-González and colleagues [23] who showed that febrile convulsions, single seizure type, and nocturnal attacks as important diagnostic hallmarks of true-DRE. The study also, showed that all cases of true-DRE had focal onset semiology. TLE was the commonest by FLE, OLE, and lastly insular lobe epilepsy. These results agreed with Hintz and colleagues [22] who found that the largest proportion of focal DRE was TLE followed by FLE.

Long-term video EEG monitoring is the gold-standard investigation for DRE diagnosis with high accuracy of syndromic epilepsy classification, interictal discharge localization, and differentiation of true-DRE from NEEE
[24]. The current study showed that long-term EEG video record is a highly sensitive procedure for diagnosis of true-DRE and identification of the SOZ but must be interpreted in the context of SSA analysis due to the possible epileptiform abnormalities in few patients with PNES with consecutive inclusion in the zone of DRE. The study also showed that patients with PNES had high incidences of ictal events during EEG recording which was very helpful in recognition of their functional seizure properties by the absence of pre-ictal $\mathrm{SOZ}$ activities and lack of alteration in the background activities before and after the ictus. These results agreed with the works of Cox and colleagues [25] as well as Elwan and colleagues [12] who showed that a valuable proportion of PNES patients had interictal EEG abnormalities attributed to abnormal functional connectivity density in frontal lobe cortex, sensorimotor cortex, cingulate gyrus, insula, and occipital cortex which could be evaluated by functional connectivity density mapping.

The study showed that 3-T MRI followed the epilepsy imaging protocols succeeded to identify structural epileptiform abnormalities in a valuable proportion of true-DRE patients with non-lesional 1.5-T MRI, yet one-third of true-DRE patients still had normal 3-T MRI. The most common structural abnormalities included MTS and FCDs. These results are in harmony with that of Duncan colleagues [26] who stated that cortical developmental abnormalities and neuronal architectural malformations including are the most common structural MRI abnormalities in the context of true-DRE cases.

Regarding 18-FDG-PET scan, the study revealed its high sensitivity for EZL in true-DRE patients where it showed regional brain hypometabolism congruent with the SOZ in $87.5 \%$ of 3 -T MRI non-lesional patients. These results are in harmony with that of Capraz and 
colleagues [27] as well as Desarnaud and colleagues [28] who identified that interictal 18-FDG-PET scan is the most sensitive procedure for localization of TLE as well as $\mathrm{SOZ}$ of extratemporal origin even for MRI-negative patients.

The multimodality patients' approach in this work revealed that all included patients with true-DRE had focal-onset seizures with TLE localization was the commonest SOZ followed by FLE, multifocal EZL, OLE, and, lastly, insular lobe epilepsy. At the same time, some patients with FLE/PET scan hypometabolism had TLElike semiology possibly due to rapid propagation of the epileptic activities from the orbital frontal surface to the temporal lobe. These results are concordant with that of Elwan and colleagues [12] who studied retrospectively concordance values of variable diagnostic tools in presurgical evaluation workup for patients with DRE and correlated these results with rate of seizure freedom after epilepsy surgery as an indicator of accurate EZL and concluded that SSA had a high lateralizing and localizing value especially when combined with interictal as well as ictal scalp EEG, MRI, and PET scan.

\section{Conclusion}

Patients with DRE need holistic multimodality assessments including SSA, long-term video EEG, high field strength MRI, and 18-FDG-PET scan to confirm the diagnosis of true-DRE, localize the $\mathrm{SOZ}$, and avoid possible misdiagnosis of NEEE.

\begin{abstract}
Abbreviations
AEDs: Antiepileptic drugs; DRE: Drug-resistant epilepsy; EZL: Epileptogenic zone localization; FCD: Focal cortical dysplasia; FDG: Fluorodeoxyglucose; FLE: Frontal lobe epilepsy; HAS: Hamilton Anxiety Scale; MADRS: Montgomery-Åsberg Depression Rating Scale; NEEE: Non-epileptic episodic events; MTS: Mesial temporal sclerosis; OLE: Occipital lobe epilepsy; PNES: Psychogenic non-epileptic seizure; PSG: Polysomnogram; SOZ: Seizure onset zone; SSA: Seizure semiology analysis; T: Tesla; TLE: temporal lobe epilepsy
\end{abstract}

\section{Acknowledgements}

We would like to thank Prof. Dr/Ehab Shawky Mohamed and Prof. Dr/Yasser Abu-Elfotouh Elheneedy for their great help in seizure semiology analysis and long-term EEG interpretation.

\section{Consent of publication}

Not applicable.

\section{Authors' contributions}

AANE: participated in the study's design, patients' selection and examination, statistical and data analysis, EEG interpretation, reference collection, and manuscript writing. WSB: participated in the study's idea, design, patients' selection and examination, statistical and data analysis, EEG and imaging interpretation, references collection, and manuscript revision. EAT: participated in the study's idea, design, patients' selection, statistical and data analysis, EEG and imaging interpretation, references collection, manuscript revision, and final approval. HAF: put the study's idea, design, patients' selection, data analysis, EEG and imaging interpretation, references collection, manuscript revision, and final approval. AAES: participated in the study's design, patients' assessment, imaging interpretation including MRI brain and PET scans, data analysis, and manuscript writing and revision. All authors read and approved the final manuscript.

\section{Funding}

No funding had been received.

\section{Availability of data and materials}

The datasets used and/or analyzed during the current study are available from the corresponding author on reasonable request.

\section{Ethics approval and consent to participate}

The manuscript was approved from The Research Ethics Committee and Quality Assurance Unit, Faculty of Medicine, Tanta University.

The URL: http://tqac.tanta.edu.eg/new-tqac/

QualityAssuranceUnit@hotmail.com

Approval Code: 30564/11/15

Name of the Pl: Abdelmoneim Ahmed Nagy Eissa.

Name of the department: Neuropsychiatry.

Type of research: MD research.

Date of approval: November 2015.

The study's protocol was approved by The Research Ethics Committee and Quality Assurance Unit, Faculty of Medicine, Tanta University. Participation was voluntary, informed consents were approved by all contributors and any possible risks were clarified.

\section{Competing interests}

The authors declare that they have no competing interests.

\section{Author details}

${ }^{1}$ Department of Neuropsychiatry, Faculty of Medicine, Tanta University, 31527 Tanta, Egypt. ${ }^{2}$ Department of Diagnostic Radiology, Faculty of Medicine, Tanta University, Tanta 31527, Egypt.

Received: 4 May 2019 Accepted: 26 August 2019

Published online: 05 September 2019

\section{References}

1. Glauser T, Ben-Menachem E, Bourgeois B, Cnaan A, Guerreiro C, Kälviäinen $\mathrm{R}$, et al. Updated ILAE evidence review of antiepileptic drug efficacy and effectiveness as initial monotherapy for epileptic seizures and syndromes. Epilepsia. 2013;54(3):551-63. https://doi.org/10.1111/epi.12074.

2. Téllez-Zenteno JF, Hernández-Ronquillo L, Buckley S, Zahagun R, Rizvi S. A validation of the new definition of drug-resistant epilepsy by the International League Against Epilepsy. Epilepsia. 2014;55(6):829-34. https:// doi.org/10.1111/epi.12633.

3. Sisterson ND, Wozny TA, Kokkinos V, Constantino A, Richardson RM. Closedloop brain stimulation for drug-resistant epilepsy: towards an evidencebased approach to personalized medicine. Neurotherapeutics. 2019;16(1): 119-27. https://doi.org/10.1007/s13311-018-00682-4.

4. Rabie MO, Tag Eldin EA, Rashed KH, Bahnasy WS, El-Serogy HA. A study on serum levels of testosterone and prolactin hormones in male epileptic adolescents. Egypt J Neurol Psychiat Neurosurg. 2016;53(2):79-83. https:// doi.org/10.4103/1110-1083.183407.

5. Gedzelman E, LaRoche S. Long-term video EEG monitoring for diagnosis of psychogenic nonepileptic seizures. Neuropsychiatr Dis Treat. 2014;10:1979. https://doi.org/10.2147/NDT.S49531.

6. Yamazoe T, Ellenrieder N, Khoo HM, Huang YH, Zazubovits N, Dubeau F, et al. Widespread interictal epileptic discharge more likely than focal discharges to unveil the seizure onset zone in EEG-fMRI. Clin Neurophysiol. 2019;130(4):429-38. https://doi.org/10.1016/j.clinph.2018.12.014.

7. Michel V, Mazzola L, Lemesle M, Vercueil L. Long-term EEG in adults: sleepdeprived EEG (SDE), ambulatory EEG (Amb-EEG) and long-term video-EEG recording (LTVER). Neurophysiol Clin. 2015;45(1):47-64. https://doi.org/10.1 016/j.neucli.2014.11.004.

8. Cendes F, Theodore W, Brinkmann B, Sulc V, Cascino G. Neuroimaging of epilepsy. Hand b Clin Neurol. 2016;136:985-1014. https://doi.org/10.1016/ B978-0-444-53486-6.00051-X.

9. Theodore W. Presurgical focus localization in epilepsy: PET and SPECT. Semin Nucl. 2017:47(1):44-53. https://doi.org/10.1053/j.semnuclmed.2016.09.008.

10. Eissa AAN. Long-term EEG monitoring and positron emission tomography in evaluating patients with drug resistant epilepsy. [MD Thesis] Faculty of Medicine: Tanta University; 2019. https://doi.org/10.13140/RG.2.2.12105.1 9043 
11. Kwan P, Arzimanoglou A, Berg A, Brodie M, Allen Hauser W, Mathern G, et al. Definition of drug resistant epilepsy: consensus proposal by the ad hoc Task Force of the ILAE Commission on Therapeutic Strategies. Epilepsia. 2010;51(6):1069-77. https://doi.org/10.1111/j.1528-1167.2009.02397.x.

12. Elwan $S$, Alexopoulos A, Silveira D, Kotagal P. Lateralizing and localizing value of seizure semiology: Comparison with scalp EEG, MRI and PET in patients successfully treated with resective epilepsy surgery. Seizure. 2018; 61:203-8. https://doi.org/10.1016/j.seizure.2018.08.026.

13. American Clinical Neurophysiology Society. Guideline twelve: guidelines for long-term monitoring for epilepsy. J Clin Neurophysiol. 2008;25(3):170-80. https://doi.org/10.1097/WNP.0b013e318175d472.

14. Sateia MJ. International classification of sleep disorders-third edition. Highlights and modifications. Chest. 2014;146(5):1387-94. https://doi.org/1 0.1378/chest.14-0970.

15. Cendes F, Theodore WH, Brinkmann BH, Sulc V, Cascino GD. Neuroimaging of epilepsy. Handb Clin Neurol. 2016;136:985-1014. https://doi.org/10.1016/ B978-0-444-53486-6.00051-X.

16. Varrone A, Asenbaum S, Vander Borght T, Booij J, Nobili F, Någren K, et al. EANM procedure guidelines for PET brain imaging using [18 F] FDG, version 2. Eur J Nucl Med Mol Imaging. 2009;36(12):2103-10. https://doi.org/10.1 007/s00259-009-1264-0.

17. An S, Malhotra K, Dilley C, Han-Burgess E, Valdez J, Robertson J, et al. Predicting drug-resistant epilepsy - a machine learning approach based on administrative claims data. Epilepsy Behav. 2018;89:118-25. https://doi.org/1 0.1016/j.yebeh.2018.10.013.

18. Wilkins S, Mesraoua B, Palomo G, Al Hail H, Salam A, Melikyan G, et al. Characteristics of patients with confirmed epilepsy and psychogenic nonepileptic seizures in Qatar. Epilepsy Behav. 2018;85:218-21. https://doi. org/10.1016/j.yebeh.2018.06.014.

19. Hanrahan B, Ghearing G, Urban A, Plummer C, Pan J, Hendrickson R, et al. Diagnostic accuracy of paroxysmal spells: clinical history versus observation. Epilepsy Behav. 2018;78:73-7. https://doi.org/10.1016/j.yebeh.2017.09.001.

20. Korucuk M, Gazioglu S, Yildirim A, Karaguzel E, Velioglu S. Semiological characteristics of patients with psychogenic nonepileptic seizures: genderrelated differences. Epilepsy Behav. 2018;89:130-4. https://doi.org/10.1016/j. yebeh.2018.10.032

21. Labate A, Mumoli L, Curcio A, Tripepi G, D’Arrigo G, Ferlazzo E, et al. Value of clinical features to differentiate refractory epilepsy from mimics: a prospective longitudinal cohort study. Eur J Neurol. 2018;25(5):711-7. https://doi.org/10.1111/ene.13579.

22. Hintz M, Krenz V, Schulze-Bonhage A. Age-dependent semiology of frontal lobe seizures. Epilepsy Res. 2019;149:83-7. https://doi.org/10.1016/j. eplepsyres.2018.10.007.

23. Guevara-González J, Dimas-Rendón I, de Guevara L, Guevara-Campos J, Cauli O. Febrile seizure and related syndromes. Neurol Psychiatry Brain Res. 2018;27:1-5. https://doi.org/10.1016/j.npbr.2017.11.003.

24. Hopp JL. Nonepileptic episodic events. Continuum (MINNEAP MINN). 2019; 25(2, EPILEPSY):492-507.

25. Cox F, Visser G. What is a practical duration time for capturing psychogenic non-epileptic seizures by video-EEG monitoring? Seizure. 2018;56:110. https://doi.org/10.1016/j.seizure.2018.02.008.

26. Duncan J, Winston G, Koepp M, Ourselin S. Brain imaging in the assessment for epilepsy surgery. Lancet Neurol. 2016;15(4):420-33. https://doi.org/10.1 016/S1474-4422(15)00383-X.

27. Capraz I, Kurt G, Akdemir Ö, Hirfanoglu T, Oner Y, Sengezer T, et al. Surgical outcome in patients with MRI-negative, PET-positive temporal lobe epilepsy. Seizure. 2015;29:63-8. https://doi.org/10.1016/j.seizure.2015.03.015.

28. Desarnaud S, Mellerio C, Semah F, Laurent A, Landre E, Devaux B, et al. ${ }^{18} \mathrm{~F}-$ FDG PET in drug-resistant epilepsy due to focal cortical dysplasia type 2: additional value of electroclinical data and coregistration with MRI. Eur J Nucl Med Mol Imaging. 2018;45(8):1449-60. https://doi.org/10.1007/s00259018-3994-3.

\section{Publisher's Note}

Springer Nature remains neutral with regard to jurisdictional claims in published maps and institutional affiliations.

\section{Submit your manuscript to a SpringerOpen ${ }^{\circ}$ journal and benefit from:}

- Convenient online submission

- Rigorous peer review

- Open access: articles freely available online

- High visibility within the field

- Retaining the copyright to your article

Submit your next manuscript at $\boldsymbol{\nabla}$ springeropen.com 